\title{
Variations in Hedgehog signaling: divergence and perpetuation in Sufu regulation of Gli
}

\author{
Laurent Ruel and Pascal P. Thérond ${ }^{1}$ \\ Institut Biologie du Développement et Cancer-IBDC, Université de Nice-Sophia Antipolis, CNRS UMR 6543, Centre de \\ Biochimie, 06108 Nice Cedex 02, France
}

The Hedgehog (Hh) proteins play a universal role in metazoan development. Nevertheless, fundamental differences exist between Drosophila and vertebrates in the transduction of the $\mathrm{Hh}$ signal, notably regarding the role of primary cilia in mammalian cells. In this issue of Genes \& Development, Chen and colleagues (pp. 1910-1928) demonstrate that mouse Suppressor of fused (Sufu) regulates the stability of the transcription factors Gli2 and Gli3 by antagonizing the conserved Gli degradation device mediated by Hib/Spop in a cilia-independent manner.

The Hedgehog (Hh) family of secreted proteins is involved in the developmental regulation of numerous tissues and organs. Initially identified in Drosophila (NüssleinVolhard and Wieschaus 1980), where it participates in the organization of the embryonic segments and adult appendages-such as wings and legs-Hh's role as an organizing molecule is highly conserved through evolution. One of its vertebrate counterparts, Sonic Hedgehog (Shh), has been studied extensively for its significant role in neural tube and limb patterning (McMahon et al. 2003). Dramatic consequences ensue from the deregulation of the Hh pathway either during development or adult life. For instance, a decrease in Shh signaling in the mammalian embryos led to cases of holoprosencephaly (failure of separation of the cephalic lobes associated with facial deformities). The recent broad interest in the Hh field certainly arose from the observation that postnatal uncontrolled pathway activation cause tragic effects: Mutations in the components of the Hh signaling pathway have been involved in the majority of cases of basal cell carcinoma, the most common cancer in humans. In addition to skin cancer, unrestrained pathway activation has been linked to the initiation or maintenance of numerous cancers, including medulloblastomas, chronic

[Keywords: Hedgehog; signal transduction; evolution; primary cilium; Sufu; Gli]

${ }^{1}$ Corresponding author.

E-MAIL therond@unice.fr; FAX 33-492076405.

Article is online at http://www.genesdev.org/cgi/doi/10.1101/gad.1838109. myelogenous leukaemia, and multiple myeloma, as well as cancers affecting the pancreas and digestive tract (Beachy et al. 2004).

The importance of $\mathrm{Hh}$ as an organizing molecule is also apparent in the conservation of its signaling pathway. In both Drosophila and vertebrates, secreted $\mathrm{Hh}$ binds to its receptor, Patched (Ptc), and to its coreceptor, interference hedgehog (ihog/Cdo). It has been proposed that Ptc, which constitutively represses the Hh pathway, belongs to a proton gradient-driven transporter family and enzymatically inhibits the conserved transmembrane protein Smoothened (Smo) of the G-protein-coupled receptor (GPCR) family (Taipale et al. 2002; Corcoran and Scott 2006). In Drosophila, the binding of $\mathrm{Hh}$ to Ptc induces translocation of Smo to the plasma membrane, leading to the activation of Smo and of the downstream zinc finger transcription factors of the Gli protein family (McMahon et al. 2003). Whereas Drosophila has only one Gli homolog-named Cubitus interruptus (Ci)-multiple Gli transcription factors (Gli1, Gli2, and Gli3) interplay in the vertebrate Hh signaling pathway. Gli3 is processed efficiently into a repressor form (GliR), whereas Gli2 contributes largely as a primary activator (GliA). Glil is a transcriptional target of the pathway and acts as a transcriptional activator that reinforces GliA. In all species, activation of $\mathrm{Hh}$ signaling inhibits proteolytic processing of the Ci/Gli cytoplasmic proteins, and leads to the production of the transcriptional activator forms. The change in the balance between the activator and the repressor forms of the Gli proteins is of major importance in the cellular responses to Hh signal and is manifested as a combination of transcriptional derepression and activation (Ruiz i Altaba et al. 2007).

\section{Important divergences in the $\mathrm{Hh}$ pathway}

Despite the apparent conservation of the fly and mammalian Hh pathways, there are important divergences concerning the relative contribution of conserved signaling components. In Drosophila, the Hh transduction is mediated by the interaction of Smo with a cytoplasmic signaling complex that includes the serine-threonine kinase Fused (Fu), the kinesin-like Costal-2 (Cos2), the 
Suppressor of Fused (Sufu) protein, and Ci (Lum et al. 2003; Ruel et al. 2003). This protein complex regulates the subcellular localization and processing of $\mathrm{Ci}$. In nonactivated cells, the direct interaction of Cos 2 with $\mathrm{Ci}$ is essential for targeting Ci for a proteasome-mediated proteolytic cleavage that generates the CiR form (see Fig. 1; Zhang et al. 2005). In cells under Hh influence, the activation of Smo leads to the phosphorylation of Cos2 by $\mathrm{Fu}$, which antagonizes the negative function of Cos2 by alleviating the cytoplasmic sequestration and processing of Ci (Ruel et al. 2007). Consequently, Ci protein is transformed into a transcriptional activator (CiA) through an unknown mechanism.

In Drosophila, Sufu loss-of-function mutations were identified by their ability to suppress the lack of $\mathrm{Fu}$ phenotype (Preat 1992) but, intriguingly, Sufu-null mutant flies are viable, suggesting that Sufu is dispensable for Hh signaling. It nevertheless has been shown that restriction of $\mathrm{Ci}$ activity when $\mathrm{Ci}$ proteolysis is blocked is due to direct binding of Sufu on Ci (Smelkinson et al. 2007), suggesting that Sufu might act as an ultimate gatekeeper to prevent $\mathrm{Ci}$ nuclear translocation when other parallel inhibitory mechanisms are defective. But the mechanism by which Sufu inhibits Ci is still poorly understood and may involve not only regulation of $\mathrm{Ci}$ subcellular localization, but also $\mathrm{Ci}$ protein level and transcriptional activity in the nucleus /Ohlmeyer and Kalderon 1998; Méthot and Basler 2000; Wang et al. 2000). Nevertheless, based on the absence of phenotype in flies, it has been proposed previously that Sufu protein has a minor role in Hh transduction (Preat 1992).

In contrast, in mice, loss of Sufu function induced ventralization of the neural tube, characteristic of a constitutive activation of the $\mathrm{Hh}$ pathway (Cooper et al. 2005; Svärd et al. 2006). This phenotype resembles the phenotype displayed by absence of Ptc, another strong repressor of the pathway. Previous work by Chuang's laboratory and others (Chen et al. 2005; Merchant et al. 2005) led to the unexpected finding that loss of mouse Fu has no consequence on $\mathrm{Hh}$ signaling in mice. Furthermore, it has been demonstrated that the closest homologs of Drosophila Cos2-kif7 and kif27-are not involved in Shh signaling, at least in mammalian fibroblast cultured cells (Varjosalo et al. 2006). Thus, the three proteins Fu, Cos2, and Sufu that provide the cytoplasmic core of the pathway in Drosophila are conserved in mammals; however, the mode of transduction has diverged.

How could such conserved proteins have diverged? This divergence might be linked to the presence of a cellular compartment, the nonmotile primary ciliumpresent on most vertebrate cells (and not fly cells) - that is necessary for Shh signaling (Fig. 1). Indeed, the lack of the intraflagellar transport complex (IFT proteins)involved in the assembly and activity of cilia through evolution-induces a defect in Shh activity, notably for the Shh-dependent ventralization of the mammalian neural tube. Colocalization of Shh and Ptc at primary cilia reinforces the idea that cilia might function as chemosensors for the detection of extracellular Shh (Rohatgi et al. 2007). In addition, the presence of cilia correlates with Hh responsiveness. Indeed, cultured cells become Shh-responsive only at confluence, and cilia are detected only during this phase (Huangfu and Anderson 2006). The fact that Drosophila does not possess such a structure and that most $\mathrm{Hh}$ mammalian components analyzed by several laboratories are localized to the primary cilium led to the proposal that primary cilia provide a new cellular function that replaces the mode of action of the Drosophila cytoplasmic core (Cos2/Fu) of the Hh pathway (see Fig. 1). Indeed, IFT proteins transport cargo along the axoneural microtubules of the cilia and present an anterograde or retrograde motor activity, which are required for both GliA function and GliR formation. This led to the following model: IFT proteins might have an instructive role in the pathway and could provide a specific assembly site for components of the $\mathrm{Hh}$ pathway or participate in transporting key players of the signaling pathway within the primary cilium to provide full activation of the pathway. Smo relocalization and regulation in primary cilium upon pathway activation support this model (Rohatgi et al. 2009). The aim of the recent study from Chuang's laboratory (Chen et al. 2009) is to understand how mammalian Sufu functions independently of Fu and Cos2 and whether it requires cilia to regulate Gli function. By using mouse embryonic fibroblasts (MEFs) derived from knockout animals and mouse genetic epistatic experiments, Chen et al. (2009) provide several major contributions, which are discussed below.

\section{A new role of mammalian Sufu in controlling Gli protein stability}

It has been shown previously that overexpressed Gli protein localizes to the primary cilium. For the first time, using specific antibodies against Gli2 and Gli3 proteins, Chen et al. (2009) document the dynamic changes in endogenous Gli localization in MEFs and show that their levels in the cilium increased upon Shh pathway activation. Sufu is also present at the primary cilium, but its location is not regulated by the pathway. Interestingly, in MEFs derived from Sufu ${ }^{-1-}$ animals, ciliary localization of Gli2 and Gli3 is abolished. But it is unlikely that Sufu regulates Gli location, because ciliary location of Glis can be rescued just by increasing their expression in $\mathrm{Sufu}^{-1-}$ MEFs. Interestingly, the lack of Gli ciliary location in the absence of Sufu correlates with an overall decrease of the endogenous level of Gli2 and Gli3 (both full-length and repressor forms), revealing a new role of mammalian Sufu in controlling Gli protein stability.

Does Sufu regulate Gli level and activity within the primary cilium? Three lines of evidence lead to a resounding "no." First, Chuang's laboratory (Chen et al. 2009) used MEF cells lacking primary cilium (in which the IFT gene Kif3A has been knocked down), and show that Sufu regulation of Gli stability is independent of the primary cilium. Second, they performed epistatic analysis between $\mathrm{Kif}_{3} \mathrm{a}^{-/-}$mutant mice (in which Hh signaling in the neural tube is reduced) and Sufu-deficient mice (in which the pathway is constitutively activated). The phenotype of $\mathrm{Sufu}^{-1-} \mathrm{Kif3}^{-/-}$embryos is similar to the 
Sufu regulation of Gli

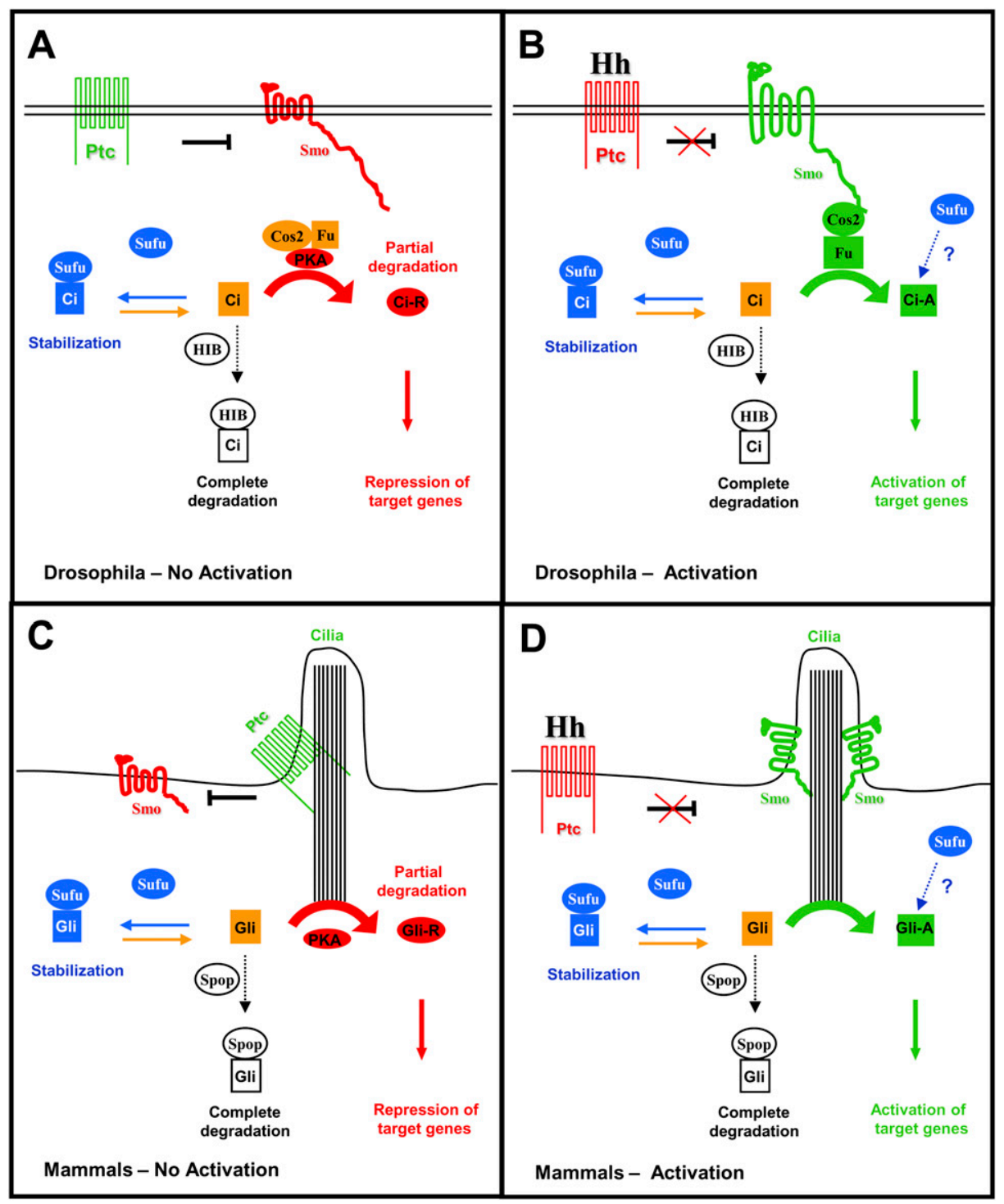

Figure 1. Stabilization, degradation, and activation of the Ci/Gli proteins by the Hh signaling pathway. (A) In Drosophila, the absence of Hh allows Ptc to inhibit Smo activity, and full-length $\mathrm{Ci}$ (Ci) activity is regulated by three different mechanisms, including phosphorylation, proteolysis, and stabilization. First, phosphorylation of $\mathrm{Ci}$ by multiple kinases, including PKA, targets Ci to the ubiquitin/proteasome-mediated partial proteolysis to generate a truncated repressor form of $\mathrm{Ci}$ (Ci-R). This step requires the kinesinlike protein Cos2, which acts as a molecular scaffold to bridge $\mathrm{Ci}$ and the kinases. Second, the activity of full-length Ci is also blocked by stoichiometric binding of Sufu, but is protected from Hib-dependent degradation. Third, the excess of Ci is controlled by Hib protein, which binds and completely degrades the full-lengh form of Ci. $(B)$ Binding of Hh to Ptc stimulates Smo phosphorylation, which increases Smo cell surface concentration. The activation of Smo stimulates the associated Fu kinase activity and dissociates Ci from the Cos 2 complex. This blocks PKA phosphorylation of $\mathrm{Ci}$ and subsequent processing. The Fu/Cos 2 complex is also necessary to form the active $\mathrm{Ci}(\mathrm{Ci}-\mathrm{A})$, but the mechanism of this activation, as its regulation by Sufu, is uncertain. (C) In mammals, in the absence of Hh, Ptc is present in the primary cilium and prevents Smo ciliary accumulation. PKA and others kinases phosphorylate Gli and target it for partial degradation to form the truncated repressor form of Gli (Gli-R). It is possible that the scaffolding role of Cos2 in Drosophila might have been replaced by the primary cilia. Independently of cilia, in the cytoplasm, the activity of full-length Gli is further blocked by Sufu, which appears to be the major mechanism for restricting Gli activity in mammals. In addition, Sufu protects Gli2 and Gli3 (but not Gli1) from degradation by Spop, the Hib mammalian homolog. (D) Binding of Hh to Ptc promotes the accumulation and activation of Smo in the cilium. Gli phosphorylation and processing are inhibited, and formation of the transcriptionally activated Gli (Gli-A) is triggered. This activation occurs in the cilia, but can also take place when both primary cilia and Sufu are lacking. The mechanism of Gli activation and Sufu regulation by Shh are not known. In both Drosophila and mammals, Sufu likely plays an important role in the nucleus, not indicated here. 
one displayed by Sufu ${ }^{-/-}$embryos. Accordingly, Gli2 and Gli3 protein levels are similarly reduced in Sufu ${ }^{-/-}$MEFs or in Kif3a $\mathrm{a}^{-1-}$ MEFS treated with shRNA for Sufu. Similar data were obtained with $\mathrm{Sufu}^{-1-}$ MEFs expressing a dominant-negative $(\mathrm{dn})$ form of another IFT protein, Kif3b. Third, overexpression of Sufu is able to suppress Gli-mediated Hh activation similarly in both wild-type MEFs and Kif3a $\mathrm{a}^{-1-}$ MEFs. Conclusions from Chuang's laboratory (Chen et al. 2009) nicely confirm a very recent similar approach developed by Jia et al. (2009), which showed that Sufu binding to and repression of Glis activity is observed in MEFs derived from IFT88-deficient embryos in which cilia formation is also disrupted. The Sufu/Ift88 double-mutant embryos are also morphologically similar to the Sufu single mutant. Taken collectively, these data suggest that regulation of Gli protein level by Sufu can occur in the absence of cilia.

Based on these results, one could wonder: For which level of pathway activation is the primary cilium necessary then? Chen et al. (2009) adequately show that the elevated $\mathrm{Hh}$ signaling in $\mathrm{Ptch} 1^{-/-}$MEFs is reduced by $80 \%$ when dnKif $3 \mathrm{~b}$ is expressed. If we assume that in this later experiment ciliary function is defective, it clearly shows that, as for Smo, Ptch1 function necessitates an intact primary cilium, but this is likely upstream of Sufu regulation of Glis.

\section{Regulation of Gli protein stability by Sufu is conserved through evolution}

If cilium integrity is not necessary for Sufu regulation of Glis, then one could hypothesize that the introduction of a form of vertebrate Sufu that does not localize to the cilium into Sufu ${ }^{-/-}$MEFs would restore Gli levels. Such Sufu variants are not available currently; however, to address this question, Chen et al. (2009) chose to manipulate Sufu from different species to challenge the conservation of this process. They show nicely that the ciliumindependent regulation of Gli stability by Sufu seems to be evolutionarily conserved-zebrafish or Drosophila versions of Sufu can rescue stability, cilia localization, and activity of Gli2 and Gli3 in MEF cells lacking Sufu activity. Since neither zebrafish nor Drosophila versions of Sufu localize to cilia, it is likely that their rescue is independent of cilia regulation. To investigate the differences in ciliumdependent processes within Hh signal transduction, Chen et al. (2009) also assayed ciliary localization of Smo from different species in $\mathrm{Smo}^{-1-}$ MEFs and show that zebrafish Smo localized to the cilium and rescued $\mathrm{Hh}$ signaling when MEFs are treated with $\mathrm{Hh}$. On the contrary, Drosophila Smo failed to reach the cilium, and no $\mathrm{Hh}$ responses were observed. In a complementary experiment, it has been shown previously that mouse Smo cannot compensate for the lack of Drosophila Smo in insect cultured cells (De Rivoyre et al. 2006). This makes sense since primary cilium regulation of the Hh signaling pathway has not been implemented in invertebrates and represents an evolutionary divergence.

The divergence downstream from Smo is still not well understood, and the precise role of vertebrate Fu and Cos2 is controversial (Tay et al. 2005; Varjosalo et al. 2006). Two homologs of Cos2-Kif7 and Kif27-have been identified in mammals, whereas only one is present in zebra fish (Kif7). This suggests that duplication of ancestral Cos2 in the vertebrate lineage might have occurred after emergence of the fish lineage (Wilson et al. 2009). Interestingly, morpholino knockdown experiments indicate that zebrafish Kif7 acts like Cos2: as a negative regulator of the Hh pathway (Tay et al. 2005). However, no mouse knockout of Kif7 has been developed yet to test for the conserved activity of Cos 2 in mouse. Another surprising observation from Chuang's laboratory (Wilson et al. 2009) was the demonstration that zebrafish $\mathrm{Fu}$ is not only necessary to control Hh signaling, but is also essential for construction of the central pair apparatus of motile cilia, which is structurally different from the primary cilium. Wilson et al. (2009) showed that, in the $\mathrm{Fu}^{-/-}$mouse, cells form primary cilia normally and $\mathrm{Hh}$ signaling is not affected, but motile ciliogenesis is. Despite this nonessential role of $\mathrm{Fu}$ in mammalian $\mathrm{Hh}$ signaling, it retains a physical association with Kif27. Thus, divergence within the Hh pathway between fish and mice might have led to a partitioning of functionality for $\mathrm{Fu}$ and Kif7, such that mouse $\mathrm{Fu}$ and Kif27 are involved in motile ciliogenesis, while a compensatory Fu kinase, associated with Kif7, is necessary for Hh signaling. This model awaits the phenotypic analysis of mice deficient in Kif7 and the kinases that have been shown to be necessary for $\mathrm{Hh}$ signaling in mammalian cells (Evangelista et al. 2008; Varjosalo et al. 2008). Alternatively, Sufu may have adopted a new function during evolution and could bypass the need for a $\mathrm{Fu} /$ kinesin pair. Indeed, the strong constitutive activation of Hh signaling in $\mathrm{Sufu}^{-/-}$mice indicates that Sufu has evolved as the major intracellular inhibitor of Gli activity (Svärd et al. 2006).

By which mechanism does Sufu regulate Gli2 and Gli3 levels? Chen et al. (2009) provide convincing data indicating that mouse Sufu regulates Gli2/3 stability by antagonizing the Spop protein of the BTB domaincontaining protein family. Indeed, Chen et al. (2009) show that Spop binds to Gli2 and Gli3 and promotes their ubiquitination and degradation in a proteasomedependent manner. The overexpression of Sufu appears to block Spop-dependent Gli protein reduction, and $\mathrm{Sufu}^{-/-}$ MEFs treated with shRNA against Spop partially rescued Gli protein levels. How conserved is this mechanism? Well, it has been shown previously that the Drosophila counterpart of Spop, the Hib protein, forms a complex with $\mathrm{Ci}$ and $\mathrm{Cul} 3$ and promotes $\mathrm{Ci}$ ubiquitination and degradation (Zhang et al. 2006). Interestingly, in the same study, it has been shown in transgenic flies that Spop can rescue Drosophila Hib-dependent degradation of $\mathrm{Ci}$, and that Gli2 and Gli3 transgenic proteins are subjected to degradation by Hib. Nevertheless, important differences in the Hib/Spop-Gli circuit exist between flies and mammals (see below).

One may wonder how the lack of Sufu-which induces a strong decrease in Gli levels-could lead to a constitutive activation of Hh signaling. Since binding of Sufu to 
Gli2 and Gli3 represses their positive transcriptional activity, it is likely that the absence of Sufu leads to an increase in the Gli2/3 activator state. So, even though the bulk of the Gli protein level is strongly reduced, the quantity of GliA forms may increase and contribute to the constitutive activation of the pathway. It is also possible that, in absence of Sufu, GliR is less stable than the full-length Gli protein from which GliA is derived, leading to a change in the ratio between GliA and GliR, as suggested in a recent publication (Jia et al. 2009). Alternatively, since GliA reflects the collective activity of all three Gli proteins, it is possible that Gli1 may also contribute to GliA in $\mathrm{Sufu}^{-1-}$ embryos. It is worth mentioning that Gli1, unlike Gli2 and Gli3, does not appear to be subject to Spop regulation. Since Gli1 expression is subjected to GliRep activity and is induced in the Sufu mutant, Chen et al. (2009) propose that the constitutive Hh signaling activity observed in the absence of Sufu results from increased levels of Gli1, triggered by Spopmediated degradation of full-length Gli2/Gli3. The possibility that the overall level of GliA could be reduced in the absence of Sufu encouraged Chen et al. (2009) to reevaluate the role of Sufu in the Hh signaling pathway. They observed that ligand-dependent pathway activation is reduced in $\mathrm{Sufu}^{-1-}$ MEFs compared with wild-type MEFs, suggesting that Sufu is also required for maximal Hh signaling. This is contradictory to a previous study (Svärd et al. 2006) showing that Smo activation does not further induce Hh pathway activation in Sufu ${ }^{-/-}$MEFs, likely due to the fact that the pathway is already maximally activated. A detailed comparison between both studies will be needed to resolve this issue.

So, if not in the cilia, in which subcellular compartment would Sufu regulate Gli protein activity? This is still an open question, but regulation likely occurs at multiple levels, since Sufu is present in both the nucleus and cytoplasm in different cell types. Based on overexpression studies, it has been proposed that the role of Sufu in both flies and mammals is to retain $\mathrm{Ci} / \mathrm{Gli}$ proteins in the cytoplasm, thereby preventing their nuclear translocation (Kogerman et al. 1999; Méthot and Basler 2000). Thus, one would expect to witness increased levels of Gli nuclear accumulation in the absence of Sufu. However, constitutive activation of Hh signaling observed in Sufu ${ }^{-1-}$ MEFs is not accompanied by an increase of nuclear Gli1 (Svärd et al. 2006). Although conclusions based on Glil studies may not be applicable to Gli2 and Gli3 given their distinct properties, the main role of Sufu in mammals might be to mediate repression of Gli-dependent transcription directly in the nucleus. Indeed, physical interaction between Sufu and Gli2/3 is not affected by Hh signaling activation, and Sufu shuttles with Ci/Gli to form a complex with nuclear Gli bound to DNA (Kogerman et al. 1999; Sisson et al. 2006). Furthermore, it has been proposed that Sufu can repress Glimediated transcription by recruiting the Sin3-histone deacetylase (HDAC) corepressor complex to promoters containing Gli-binding elements (Cheng and Bishop 2002). In the present study, Chen et al. (2009) show that Spop has both cytoplasmic and nuclear localization, and that both nuclear and cytoplasmic Gli2/3 protein levels are reduced in the absence of Sufu. So, it is conceivable that Sufu controls full-length Gli protein levels in the cytoplasm but also protects Gli proteins in the nucleus, where Hib/Spop proteins are present.

In the Drosophila wing imaginal disc, Hib expression depends on Hh signaling. Moreover, in eye imaginal discs, where Hh signals coordinate cell proliferation and differentiation, Hib is highly expressed in the differentiating cells to prevent aberrant $\mathrm{Hh}$ signaling activity and ensure normal eye development (Zhang et al. 2006). So, it is possible that, in mammals, Hh pathway activity needs to be turned off in differentiating cells to allow for their normal development, and Spop might represent a mechanism to terminate $\mathrm{Hh}$ signaling, as is the case in Drosophila eye development. It thus will be interesting to know whether Spop expression depends on Hh signaling in mouse embryonic development, and whether the association of Spop with Glis depends on the state of the Hh pathway. In both flies and mammals, defects of Ci/Gli degradation mechanisms potentiate the ability of $\mathrm{Ci} / \mathrm{Gli}$ proteins to induce tissue overgrowth or tumor formation. Future studies should determine whether Spop acts as tumor suppressor and whether it contributes to Glidependent tumor formation. So, although important differences in Hh signaling between flies and mammals exist, as illustrated with the role of the primary cilium, the level of conservation of $\mathrm{Hh}$ signaling between species is still a topical question.

\section{Acknowledgments}

We thank Katie Ayers and Rune Tofgard for helpful comments on the manuscript. Our research is supported by grants from La Ligue Nationale Contre Le Cancer "Equipe labellisée 2008."

\section{Note added in proof}

During the proof correction of this perspective, two studies related the knockout of the kinesin Kif7 in mice showing that Kif7 is a critical regulator of Hh signaling in vivo (Cheung et al. 2009; Endoh-Yamagami et al. 2009).

\section{References}

Beachy PA, Karhadkar SS, Berman DM. 2004. Tissue repair and stem cell renewal in carcinogenesis. Nature 432: 324331.

Chen MH, Gao N, Kawakami T, Chuang PT. 2005. Mice deficient in the fused homolog do not exhibit phenotypes indicative of perturbed hedgehog signalling during embryonic development. Mol Cell Biol 25: 7042-7053.

Chen M-H, Wilson CW, Li Y-J, Law KKL, Lu C-S, Gacayan R, Zhang X, Hui C-c, Chuang P-T. 2009. Cilium-independent regulation of Gli protein function by Sufu in Hedgehog signaling is evolutionarily conserved. Genes \& Dev (this issue). doi: 10.1101/gad.1794109.

Cheng SY, Bishop JM. 2002. Suppressor of Fused represses Glimediated transcription by recruiting the SAP18-mSin3 corepressor complex. Proc Natl Acad Sci 99: 5442-5447.

Cheung HO, Zhang X, Ribeiro A, Mo R, Makino S, Puviindran V, Law KK, Briscoe J, Hui CC. 2009. The kinesin protein Kif7 is a critical regulator of Gli transcription factors in mammalian 
hedgehog signaling. Sci Signal 2: ra29. doi: 10.1126/scisignal. 2000405.

Cooper AF, Yu KP, Brueckner M, Brailey LL, Johnson L, McGrath JM, Bale AE. 2005. Cardiac and CNS defects in a mouse with targeted disruption of suppressor of fused. Development 132: 4407-4417.

Corcoran RB, Scott MP. 2006. Oxysterols stimulate Sonic hedgehog signal transduction and proliferation of medulloblastoma cells. Proc Natl Acad Sci 103: 8408-8413.

De Rivoyre M, Ruel L, Varjosalo M, Loubat A, Bidet M, Therond PP, Mus-Veteau I. 2006. Human receptors patched and smoothened partially transducer hedgehog signal when expressed in Drosophila cells. I Biol Chem 281: 2858428595.

Endoh-Yamagami S, Evangelista M, Wilson D, Wen X, Theunissen JW, Phamluong K, Davis M, Scales SJ, Solloway MJ, de Sauvage FJ, et al. 2009. The mammalian Cos2 homolog Kif7 plays an essential role in modulating $\mathrm{Hh}$ signal transduction during development. Curr Biol doi: 10.1016/j.cub.2009.06.046.

Evangelista M, Lim TY, Lee J, Parker L, Ashique A, Peterson AS, Ye W, Davis DP, de Sauvage FJ. 2008. Kinome siRNA screen identifies regulators of ciliogenesis and hedgehog signal transduction. Sci. Signal 1: ra7. doi: 10.1126/scisignal.1162925.

Huangfu D, Anderson KV. 2006. Signaling from Smo to Ci/Gli: Conservation and divergence of Hedgehog pathways from Drosophila to vertebrates. Development 133: 3-14.

Jia J, Kolterud A, Zeng H, Hoover A, Teglund S, Toftgård R, Liu A. 2009. Suppressor of Fused inhibits mammalian Hedgehog signalling in the absence of cilia. Dev Biol 330: 452-460.

Kogerman P, Grimm T, Kogerman L, Krause D, Undén AB, Sandstedt B, Toftgård R, Zaphiropoulos PG. 1999. Mammalian suppressor-of-fused modulates nuclear-cytoplasmic shuttling of Gli-1. Nat Cell Biol 1: 312-319.

Lum L, Zhang C, Oh S, Mann RK, von Kessler DP, Taipale J, Weis-Garcia F, Gong R, Wang B, Beachy PA. 2003. Hedgehog signal transduction via Smoothened association with a cytoplasmic complex scaffolded by the atypical kinesin, Costal-2. Mol Cell 12: 1261-1274.

McMahon AP, Ingham PW, Tabin CJ. 2003. Development roles and clinical significance of Hedgehog signalling. Curr Top Dev Biol 53: 1-114.

Merchant M, Evangelista M, Luoh SM, Frantz GD, Chalasani S, Carano RA, van Hoy M, Ramirez J, Ogasawara AK, McFarland LM, et al. 2005. Loss of the serine/threonine kinase fused results in postnatal growth defects and lethality due to progressive hydrocephalus. Mol Cell Biol 25: 7054-7068.

Méthot N, Basler K. 2000. Suppressor of fused opposes hedgehog signal transduction by impeding nuclear accumulation of the activator form of Cubitus interruptus. Development 127: 4001-4010.

Nüsslein-Volhard C, Wieschaus E. 1980. Mutations affecting segment number and polarity in Drosophila. Nature 287: 795-801.

Ohlmeyer JT, Kalderon D. 1998. Hedgehog stimulates maturation of Cubitus interruptus into a labile transcriptional activator. Nature 396: 749-753.

Preat T. 1992. Characterization of Suppressor of fused, a complete suppressor of the fused segment polarity gene of Drosophila melanogaster. Genetics 132: 725-736.

Rohatgi R, Milenkovic L, Scott MP. 2007. Patched regulates hedgehog signalling at the primary cilium. Science 317: 372 376.

Rohatgi R, Milenkovic L, Corcoran RB, And Scott MP. 2009. Hedgehog signal transduction by Smoothened: Pharmacologic evidence for a 2-step activation process. Proc Nat1 Acad Sci 106: 3196-3201.
Ruel L, Rodriguez R, Gallet A, Lavenant-Staccini L, Therond PP. 2003. Stability and association of Smoothened, Costal2 and Fused with Cubitus interruptus are regulated by Hedgehog. Nat Cell Biol 5: 907-913.

Ruel L, Gallet A, Raisin S, Truchi A, Staccini-Lavenant L, Cervantes A, Therond PP. 2007. Phosphorylation of the atypical kinesin Costal2 by the kinase Fused induces the partial disassembly of the Smoothened-Fused-Costal2Cubitus interruptus complex in Hedgehog signalling. Development 134: 3677-3689.

Ruiz i Altaba A, Mas C, Stecca B. 2007. The Gli code: An information nexus regulating cell fate, stemness and cancer. Trends Cell Biol 17: 438-447.

Sisson BE, Ziegenhorn SL, Holmgren RA. 2006. Regulation of Ci and $\mathrm{Su}(\mathrm{fu})$ nuclear import in Drosophila. Dev Biol 294: 258270.

Smelkinson MG, Zhou Q, Kalderon D. 2007. Regulation of Ci$\mathrm{SCF}(\mathrm{Slimb})$ binding, Ci proteolysis, and Hedgehog pathway activity by Ci phosphorylation. Dev Cell 13: 481-495.

Svärd J, Heby-Henricson K, Persson-Lek M, Rozell B, Lauth M, Bergström A, Ericson J, Toftgård R, Teglund S. 2006. Genetic elimination of Suppressor of Fused reveals an essential repressor function in the mammalian Hedgehog signalling pathway. Dev Cell 10: 187-197.

Taipale J, Cooper MK, Maiti T, Beachy PA. 2002. Patched acts catalytically to suppress the activity of Smoothened. Nature 418: 892-897.

Tay SY, Ingham PW, Roy S. 2005. A homologue of the Drosophila kinesin-like protein Costal2 regulates Hedgehog signal transduction in the vertebrate embryo. Development 132: 625-634.

Varjosalo M, Li SP, Taipale J. 2006. Divergence of hedgehog signal transduction mechanism between Drosophila and mammals. Dev Cell 10: 177-186.

Varjosalo $M$, Björklund $M$, Cheng $F$, Syvänen $H$, Kivioja $T$, Kilpinen S, Sun Z, Kallioniemi O, Stunnenberg HG, He WW, et al. 2008. Application of active and kinase-deficient kinome collection for identification of kinases regulating hedgehog signaling. Cell 133: 537-548.

Wang G, Amanai K, Wang B, Jiang J. 2000. Interactions with Costal2 and suppressor of fused regulate nuclear translocation and activity of Cubitus interruptus. Genes \& Dev 14: 2893-2905.

Wilson CW, Nguyen CT, Chen MH, Yang JH, Gacayan R, Huang J, Chen JN, Chuang PT. 2009. Fused has evolved divergent roles in vertebrate Hedgehog signalling and motile ciliogenesis. Nature 459: 98-102.

Zhang W, Zhao Y, Tong C, Wang G, Wang B, Jia J, Jiang J. 2005. Hedgehog-regulated Costal2-kinase complexes control phosphorylation and proteolytic processing of Cubitus interruptus. Dev Cell 8: 267-278.

Zhang Q, Zhang L, Wang B, Ou CY, Chien CT, Jiang J. 2006. A hedgehog-induced BTB protein modulates hedgehog signaling by degrading Ci/Gli transcription factor. Dev Cell 10: 719-729. 